\title{
Retromolar Trigone
}

National Cancer Institute

\section{Source}

National Cancer Institute. Retromolar Trigone. NCI Thesaurus. Code C54223.

A triangular-shaped area of mucosa located posterior the last mandibular molar tooth. 